\title{
Comparative genomics of aldehyde dehydrogenase 5al (succinate semialdehyde dehydrogenase) and accumulation of gamma-hydroxybutyrate associated with its deficiency
}

\author{
Patrizia Malaspina,' Matthew J. Picklo, ${ }^{2}$ C. Jakobs, ${ }^{3}$ O. Carter Snead ${ }^{4}$ and K. Michael Gibson ${ }^{5 *}$ \\ 'Department of Biology, Tor Vergata University, Via della Ricerca Scientifica, I-00133 Rome, Italy \\ ${ }^{2}$ Department of Pharmacology, Physiology, and Therapeutics, University of North Dakota School of Medicine and Health Sciences, \\ 501 N Columbia Road, Stop 9037, Grand Forks, ND, 58202-9037 USA \\ ${ }^{3}$ Metabolic Unit, Department of Clinical Chemistry, PK 1X 014, VU University Medical Center, 1081 HV Amsterdam, \\ The Netherlands \\ ${ }^{4}$ Program in Neuroscience and Mental Health, Hospital for Sick Children, Toronto, Ontario, Canada, and Department of \\ Pharmacology, University of Toronto Epilepsy Research Program, Hospital for Sick Children, 555 University Avenue, Toronto, \\ Ontario M5G 1X8, Canada \\ ${ }^{5}$ Division of Medical Genetics, Departments of Pediatrics, Pathology and Human Genetics, University of Pittsburgh School \\ of Medicine and Graduate School of Public Health, Rangos Research Center, 3460 Fifth Avenue, Pittsburgh, PA, 15213 USA \\ *Correspondence to: Tel: +1 412692 7608; Fax: +1 412692 7816; E-mail: michael.gibson@chp.edu
}

Date received (in revised form): 4th August 2008

\begin{abstract}
Succinic semialdehyde dehydrogenase (SSADH; aldehyde dehydrogenase 5AI [ALDH5AI]; locus 6p22) occupies a central position in central nervous system (CNS) neurotransmitter metabolism as one of two enzymes necessary for $\gamma$-aminobutyric acid (GABA) recycling from the synaptic cleft. Its importance is highlighted by the neurometabolic disease associated with its inherited deficiency in humans, as well as the severe epileptic phenotype observed in Aldh5al $\mathrm{I}^{-/}$knockout mice. Expanding evidence now suggests, however, that even subtle decreases in human SSADH activity, associated with rare and common single nucleotide polymorphisms, may produce subclinical pathological effects. SSADH, in conjunction with aldo-keto reductase 7A2 (AKR7A2), represent two neural enzymes responsible for further catabolism of succinic semialdehyde, producing either succinate (SSADH) or $\gamma$-hydroxybutyrate (GHB; AKR7A2). A GABA analogue, GHB is a short-chain fatty alcohol with unusual properties in the CNS and a long pharmacological history. Moreover, SSADH occupies a further role in the CNS as the enzyme responsible for further metabolism of the lipid peroxidation aldehyde 4-hydroxy-2-nonenal (4-HNE), an intermediate known to induce oxidant stress. Accordingly, subtle decreases in SSADH activity may have the capacity to lead to regional accumulation of neurotoxic intermediates (GHB, 4-HNE). Polymorphisms in SSADH gene structure may also associate with quantitative traits, including intelligence quotient and life expectancy. Further population-based studies of human SSADH activity promise to reveal additional properties of its function and additional roles in CNS tissue.
\end{abstract}

Keywords: GABA, GHB, succinic semialdehyde dehydrogenase deficiency, aldehyde dehydrogenase $5 A$ I, chromosome 6p22, Aldh5al I- mouse model, alternative splicing, AKR7A2, polymorphic variation, quantitative traits 


\section{Introduction}

In mammalian brain, $\gamma$-aminobutyric acid (GABA) is quantitatively the most important inhibitory neurotransmitter, and more than 30 per cent of neurones in the central nervous system (CNS) employ it to mediate inhibitory signalling. ${ }^{1,2}$ As for other neurotransmitters, the process of release, reuptake and further metabolism is tightly controlled, tuned to ensure that excitatory/inhibitory transmission maintains equilibrium. For GABA, these processes are mediated by three enzymes: glutamic acid decarboxylase (GAD), which forms GABA from glutamate, and the degradative enzymes GABA transaminase (GABA-T) and aldehyde dehydrogenase 5A1 (ALDH5A1; succinate semialdehyde dehydrogenase [SSADH]) (Figures 1 and 2). Human disorders of GAD and GABA-T are rare to non-existent; ${ }^{3}$ conversely, numerous patients with ALDH5A1 deficiency have been identified since the description of the index patient. ${ }^{4-6}$ Patient detection was facilitated by the description of $\boldsymbol{\gamma}$-hydroxybutyrate (GHB), the key biomarker for the disorder, in the urine of patients using combined gas chromatography mass spectrometry (GCMS). Accordingly, human ALDH5A1 deficiency is a rare neurometabolic disorder in which two neuromodulatory species, namely GABA and GHB, accumulate in patients' physiological fluids. ${ }^{6}$

In recent years, an emerging body of literature has revealed that ALDH5A1 may have physiological roles and functions not previously recognised. The location of the ALDH5A1 gene on chromosome $6 \mathrm{p} 22$ has led to an ongoing examination of its role in cognition and other processes. For example, the 6 p22 locus has been implicated in a number of quantitative traits, including cognitive decline, developmental dyslexia, schizophrenia and a number of epileptic syndromes. $^{7-10}$ The observation that patients with significantly decreased ALDH5A1 activity have a neurological syndrome is consistent with the involvement of ALDH5A1 in the expression of these traits. In addition, ALDH5A1 has proven to be a major enzyme involved in the metabolism of the neurotoxic lipid peroxidation product 4-hydroxy-2-nonenal (4-HNE). ${ }^{11}$ In addition, decreased ALDH5A1 activity results in significant accumulation of GABA and GHB. We do not know, however, how subtle diminutions of ALDH5A1 activity, associated with activity polymorphisms that do not induce outright disease, may lead to accumulation of neuromodulators (GABA, GHB) or toxic aldehydes (eg 4-HNE). It may be that minor accumulations of these species could contribute to some of the quantitative traits described above.

In this review, the pathophysiological traits associated with human and murine ALDH5A1 deficiency are highlighted. The pharmacology and neurotoxic aspects of GHB are described, as is the pleiotropic role of ALDH5A1 in aldehyde metabolism. Finally, the polymorphic variations in the ALDH5A1 coding region and their potential relationship with complex disease mechanisms are summarised. The goal is to provide the reader with an insight into $A L D H 5 A 1$ genomics, in both normal and diseased states.

\section{GHB}

GHB (sodium oxybate/Xyrem ${ }^{\mathrm{R}}$ ) is a well known neuromodulatory analogue of GABA. It was originally developed as a potential anaesthetic, since it could freely traverse the blood-brain barrier and reconvert to GABA, thereby exerting an inhibitory role. Early studies in animals precluded its clinical use, as pharmacological doses resulted in posture abnormalities and alterations of the electroencephalogram (EEG). ${ }^{12}$ Building upon these observations, the work of Liu and colleagues ${ }^{13}$ led to the development of GHB (and its prodrug $\gamma$-butyrolactone) as a model compound for the induction of absence in rodents.

Despite an expanding literature, it remains unclear whether or not GHB is a neurotransmitter. ${ }^{14,15}$ GHB is found in the mammalian brain at low levels, a fraction of the concentration of GABA. There are regionally located, high-affinity GHB receptors in the brain, with a well-documented developmental ontogeny. ${ }^{12,15}$ In addition, GHB is transported into the brain via a sodium- and energy-dependent gradient; is released in a calcium-dependent process; is linked to dopamine release and reuptake; and has the 


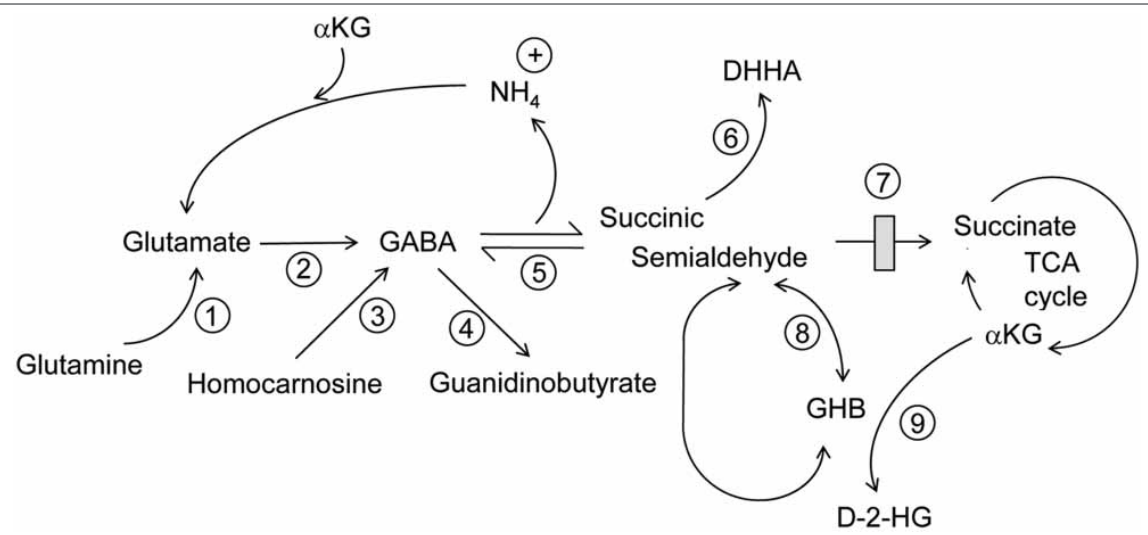

Figure I. Metabolic interrelationships of $\gamma$-aminobutyric acid (GABA) synthesis and metabolism in mammals, and metabolic abnormalities identified in murine and human aldehyde dehydrogenase 5AI (ALDH5AI) deficiency (not all steps are shown). The site of the block in ALDH5AI deficiency is indicated by the shaded box. The reader's attention is called to potential disturbances in the tricarboxylic acid (TCA) cycle induced by ALDH5AI deficiency. Note that $\alpha$-ketoglutarate ( $\alpha$-KG; also known as 2-ketoglutarate) is the nitrogen acceptor for conversion of GABA to succinic semialdehyde (SSA) (thus regenerating glutamate in the GABA shunt), and is the acceptor for hydrogen moieties in the reaction consuming $\gamma$-hydroxybutyrate (GHB), with production of D-2-hydroxyglutaric acid (D-2-HG) catalysed by D-2-hydroxyglutarate transhydrogenase (HOT). Especially in neural tissue, the TCA cycle may be depleted in $\alpha-\mathrm{KG}$, which may be further attenuated by loss of succinate from blocked GABA degradation, and the potential consumption of either pyruvate or acetyl coenzyme $A$ in the formation of 4,5-dihydroxyhexanoic acid (DHHA) from SSA. Circled numbers represent the following enzymes: I, glutaminase; 2, glutamic acid decarboxylase; 3, homocarnosinase (probably the same as carnosinase); 4, arginine:glycine amidinotransferase (putative); 5, GABA transaminase; 6, unproven reaction, but likely to involve pyruvate dehydrogenase; 7, succinate semialdehyde dehydrogenase (ALDH5AI); 8, aldo-keto reductase 7A2 (AKR7A2); 9, HOT. Note that HOT is a reversible, $\mathrm{NAD}^{+}$-independent transhydrogenase converting GHB and $\alpha$-KG to SSA and D-2-HG.

capacity to stimulate G-protein-coupled second messenger pathways. ${ }^{12}$ At pharmacological doses, GHB can induce profound EEG alterations and behavioural effects in animals and man. ${ }^{16}$

The ability of GHB to traverse the blood-brain barrier first led to its abuse potential in bodybuilders, in whom overdoses had been observed. ${ }^{17}$ Conversion of GHB to GABA, and a purported growth hormone-releasing effect of the latter, suggested the potential utility of GHB for increasing muscle mass, and, in the early 1990s, GHB was openly available. Consumption of GHB is purported to induce feelings of euphoria and disinhibition, probably mediated via the dopaminergic ventral tegmental area system, which are comparable to those associated with ethanol. ${ }^{12}$ Impairment of short-term memory may underlie the use of GHB in acquaintance sexual assault. ${ }^{18}$ Today, because of its abuse liability, GHB is a Class I controlled substance, while simultaneously being a Class III species related to its pharmacotherapeutic utility in treating cataplexy. ${ }^{19}$ Under the trade name $\mathrm{Xyrem}^{\mathrm{R}}$, GHB has also demonstrated clinical utility in fibromyalgia, alcohol dependence and post-traumatic stress disorder. ${ }^{20-22}$ Furthermore, Frucht and coworkers ${ }^{23}$ have reported clinical improvement with sodium oxybate intervention in a group of patients with ethanol-responsive myoclonus and essential tremor.

\section{The high-affinity GHB receptor, AKR7A2 and neurological disease}

As noted above, the role of GHB as an endogenous neuromodulator/neurotransmitter with its own highaffinity receptor is controversial. The sedative and hypnotic effects of $\mathrm{GHB}$ are mostly attributed to $\mathrm{GABA}_{\mathrm{B}}$ receptor signalling. However, there are regionally located, high-affinity GHB receptors in brain, with a well-documented developmental ontogeny for these receptors. ${ }^{24,25}$ Two binding sites, with high and low affinity, respectively, are found in the brain. One report using receptor binding in rat brain preparations with ${ }^{3} \mathrm{H}-\mathrm{GHB}$ indicated two sites, with affinities of 


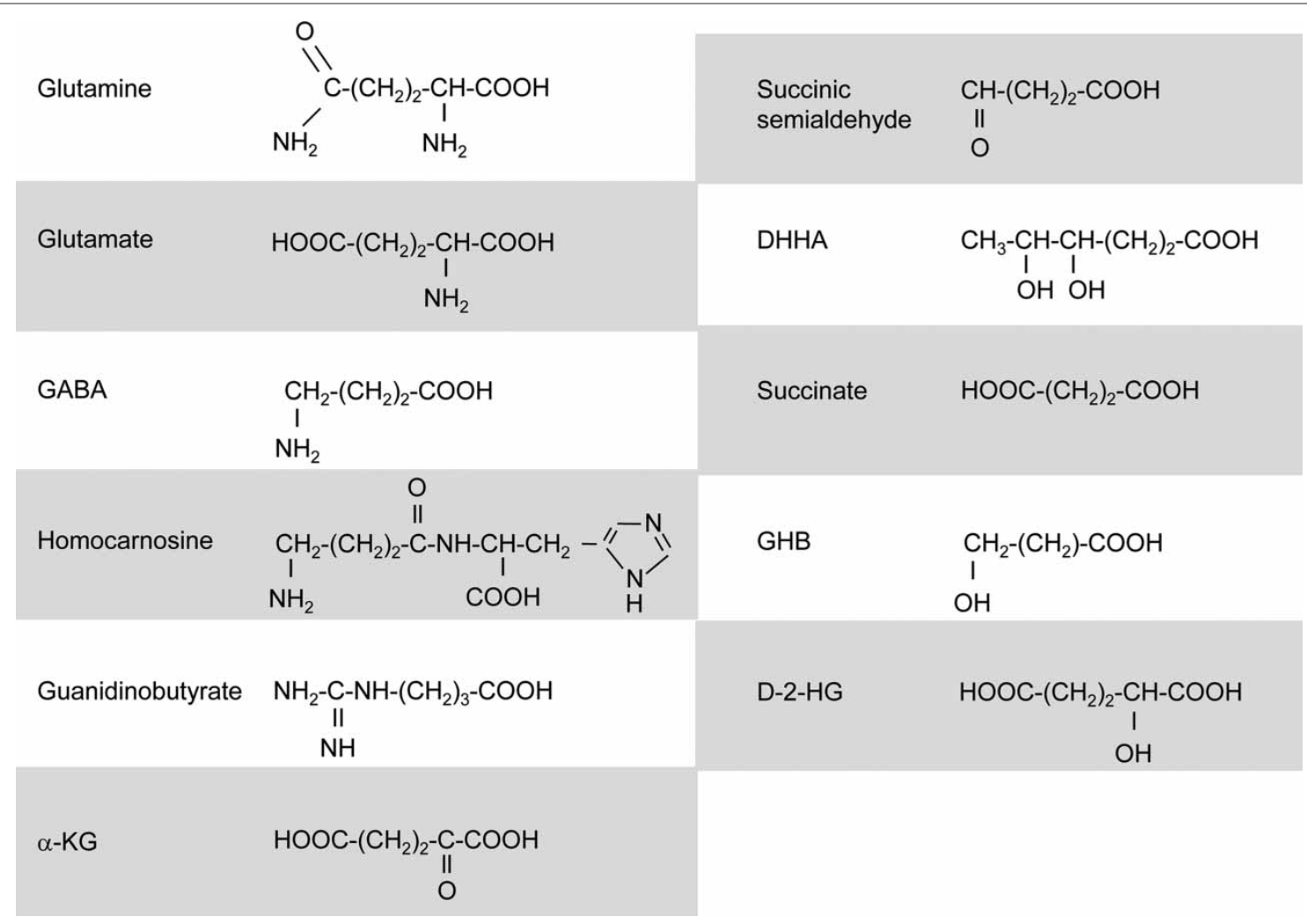

Figure 2. Corresponding chemical structures of intermediates and metabolites depicted in Figure I, and throughout the text.

$0.6 \mu \mathrm{M}$ and $2.3 \mu \mathrm{M}$, respectively. Another study, using rat brain, reported sites with affinities of $0.10 \mu \mathrm{M}$ and $16 \mu \mathrm{M}$, respectively. ${ }^{26,27}$ The reasons for these discrepancies remain unclear. Quantitative autoradiography in the human brain demonstrated two sites with dissociation constants (KD) of $0.09 \mu \mathrm{M}$ and $0.92 \mu \mathrm{M}$, respectively. ${ }^{28}$ Autoradiography studies using ${ }^{3} \mathrm{H}-\mathrm{NCS} 382$ or ${ }^{3} \mathrm{H}-\mathrm{GHB}$ indicate that binding is highest in the neuronal layers of the hippocampus and the grey matter of the cerebral cortex, with low binding observed in the cerebellum. ${ }^{28,29}$ Radioligand binding studies using ${ }^{3} \mathrm{H}-\mathrm{NCS} 382$ show that levels of the high-affinity GHB receptor increase with age (nine versus 27 weeks) in the cerebral cortex but not in hippocampus. ${ }^{30}$

Three recently cloned receptors (human and rat), that have binding characteristics similar to the highaffinity GHB receptor, were reported. ${ }^{31,32}$ The rat receptor cloned from a rat hippocampal cDNA library revealed a $\mathrm{KD}$ of $0.43 \mu \mathrm{M}{ }^{31}$ Expression of the cDNA for this putative $\mathrm{GHB}$ receptor induces depolarisation in the presence of GHB. Unexpectedly, however, the high-affinity GHB receptor antagonist NCS-382 did not block this effect. An antibody raised against the rat protein yielded similar localisation results as the autoradiographic studies. ${ }^{33}$ Two putative human receptors (GHBh1 and C12K32) were cloned from a human frontal cortex cDNA library. ${ }^{32}$ Amino acid sequence and GTP $\gamma S$ binding data indicated that both proteins were G-protein-coupled receptors. These data support previous findings that the highaffinity GHB receptor is a G-protein-coupled receptor. ${ }^{34}$ Application of submicromolar amounts of GHB stimulated membrane depolarisation in Chinese hamster ovary $(\mathrm{CHO})$ cells expressing both cDNAs, and the $\mathrm{KD}$ of the $\mathrm{C} 12 \mathrm{~K} 32$ clone for $\mathrm{GHB}$ was $0.11 \mu \mathrm{M}$.

As noted above, GHB is found in the mammalian brain at low levels $(<3 \mu \mathrm{M}),{ }^{27,35}$ indicating that $\mathrm{GHB}$ is at a sufficient concentration in vivo to bind to the high-affinity receptor. In vivo data using $\mathrm{GABA}_{\mathrm{B} 1}$ receptor-null mice, however, indicate that 
many of the physiological effects of GHB are GABA $_{\mathrm{B}}$-dependent. ${ }^{24}$ These data do not, however, rule out a neuromodulatory role for the highaffinity GHB receptor. The study of these newly cloned high-affinity $\mathrm{GHB}$ receptors will aid in clarifying the overall role of the GHB receptor.

Although multiple aldo-keto reductases (AKRs) have the potential to catalyse the reduction of SSA, AKR7A2 appears to be the main, if not only, physiologically relevant enzyme that synthesises GHB. ${ }^{36}$ As opposed to the neuronal localisation of the high-affinity GHB receptor in humans, AKR7A2 is primarily localised in astrocytes in the cerebral cortex and hippocampus. In other regions of the human brain (eg the substantia nigra and globus pallidus), neurones are immunopositive. ${ }^{37}$ Subcellularly, localisation appeared to be perinuclear and cytoplasmic in neurones and astrocytes. Although succinic semialdehyde is synthesised in mitochondria (see above), it is unclear as to why GHB synthesis would occur in cellular perinuclear regions. It is possible that AKR7A2 may also have other cellular functions.

In contrast to the glial expression of human AKR7A2, the rat isoform is expressed heavily in hippocampal neurones. ${ }^{37}$ The rat isoform was solely localised to the perinuclear region in astrocytes, although cytoplasmic staining was observed in neurones. Other studies demonstrate that the AKR7A2 isoform is also localised to various neurones in the substantia nigra. ${ }^{1}$ AKR7A2 expression is altered in human neurodegenerative disease. Probably associated with reactive gliosis, levels of AKR7A2 are elevated in the cerebral cortex of patients with Alzheimer's disease (AD). ${ }^{37}$ In addition to astrocytes, microglia immunopositive for AKR7A2 were found in patients with $\mathrm{AD}$. Interestingly, in patients with dementia with Lewy bodies (DLB), nigral Lewy bodies are immunoreactive for AKR7A2 content. ${ }^{37}$ The extent to which the changes in AKR7A2 expression in AD and DLB alter GHB neurochemistry or other cellular functions is unknown. GHB content is elevated in the caudate and substantia nigra of patients with Huntington's disease and in rodents following striatal injection of kainic acid. ${ }^{38,39}$ The extent to which the high-affinity
GHB receptor is altered in neurodegenerative diseases is not known.

\section{Human ALDH5AI deficiency}

First described in $1981,{ }^{4}$ human ALDH5A1 deficiency (SSADH deficiency; OMIM 610045; 271980) is the most frequently encountered disorder of GABA degradation. ${ }^{3}$ The phenotype is that of diffuse encephalopathy, most often as a static condition, comprising psychomotor delays, ataxia, hypotonia, dysfunctional or absent speech, and variable seizures. ${ }^{6}$ Patients are readily identified through screening for increased levels of GHB, generally detected at very low levels in human urine. Phenotypic variation is extensive (even within sibships), and there is no correlation between genotype and phenotype, suggesting that modifying genetic or epigenetic factors are of importance in disease pathology. Akaboshi and coworkers ${ }^{40}$ summarised inherited mutations, including missense, nonsense, gene deletions and splicing errors, without identification of a major mutation hotspot. Nonetheless, the loss or mutation of several well-conserved glycine residues, previously reported as critical for enzyme function, led to nearly complete ablation of enzyme activity. ${ }^{40,41}$

Since the report by Akaboshi and coworkers, ${ }^{40}$ the clinical and molecular heterogeneity associated with ALDH5A1 deficiency has been extended. Bekri and coworkers ${ }^{42}$ described a patient with a mild phenotype who was homozygous for a small exon 10 deletion. The mutation yields a polypeptide lacking the terminal 47 amino acid residues and containing five erroneous amino acids instead. Lemes and coworkers ${ }^{43}$ documented ALDH5A1 deficiency in South America. The patient's Spanish ancestry was consistent with the c.1226G $>$ A mutation identified in the Mediterranean area. ${ }^{40}$ Novel disease-causing mutations were detected in two families, in which the probands presented severe clinical features. In one, the mutation resulted in exon 7 deletion but maintenance of the reading frame. ${ }^{44}$ In the second case, a c.667T $>\mathrm{C}$ missense mutation resulted in a substitution of arginine for cysteine at position 223 of exon 4 (Malaspina and 
coworkers, unpublished). Loss of this cysteine residue has been previously demonstrated to dramatically reduce enzyme activity. ${ }^{40} \mathrm{~A}$ patient with combined ALDH5A1 deficiency and incomplete WAGR (aniridia, hemihypertrophy, and Wilms' tumour) syndrome was described by Jung and collaborators. ${ }^{45}$ Absence of enzyme activity in the proband's leukocytes was associated with homozygosity for a novel c.587G $>$ A missense mutation (p.G196D). This gene variant was not detected in numerous control DNA samples, and the glycine residue is highly conserved, suggesting pathogenicity.

Recent evidence has described a phenotype in adolescent and adult patients that comprises significant neuropsychiatric morbidity, including hallucinations, aggressive behaviour, obsessioncompulsion and similar features. ${ }^{46}$ Treatment approaches are generally symptomatic. Current therapeutic intervention is primarily limited to vigabatrin, an irreversible GABA-T inhibitor which decreases cerebrospinal fluid (CSF) GHB concentration and may lead to some improvement of ataxia, behaviour and manageability. The side effects of long-term vigabatrin administration include gastrointestinal discomfort, neurological symptoms and retinal toxicity. ${ }^{47}$ Consistent efficacy of vigabatrin treatment has been reported in the first case of paroxysmal dystonia occurring in siblings with ALDH5A1 deficiency. ${ }^{48}$ More recent findings have suggested therapeutic utility with the non-physiological amino acid taurine and the ketogenic diet employed for refractory seizures. ${ }^{49,50}$

Studies in patient physiological fluids, primarily CSF, have revealed the presence of a number of neuromodulatory compounds, including GABA and GHB. $^{51}$ GABA has three major receptors in the brain, $\mathrm{GABA}_{\mathrm{A}}, \mathrm{GABA}_{\mathrm{B}}$ and $\mathrm{GABA}_{\mathrm{C}}$ (the latter is predominantly localised to ocular tissue). $\mathrm{GABA}_{\mathrm{A}}$ mediates fast neuroinhibition linked to gated chloride channels, whereas $G_{A B A}$ acts via $G$ proteins and the adenylate cyclase system and is responsible for slower, long-term inhibitory neurotransmission. ${ }^{12} \mathrm{GHB}$, at very high (non-physiological) concentrations, has a weak affinity for the $G_{A B A}$ receptor, but no demonstrable activity at $G_{A B A}$ receptors. $^{25}$ Accumulation of GABA and GHB raises the possibility that brain receptors are functionally downregulated in the presence of excess ligand. Accordingly, recent data suggest alterations of the $\mathrm{GABA}_{\mathrm{A}}$ receptor in ALDH5A1 patients, detected using $\left({ }^{11} \mathrm{C}\right)$-flumazenil binding in vivo. ${ }^{52}$ Additionally, studies employing transcranial magnetic stimulation (TMS), which estimates endogenous GABAergic activity, have revealed $\mathrm{GABA}_{\mathrm{B}}$ receptor abnormalities in human patients. ${ }^{53}$ These functional alterations in GABAergic systems, documented in both ALDH5A1-deficient patients and mice (see below), have become the basis for clinical interventions targeting the GABA system in patients.

\section{Murine ALDH5Al deficiency}

In order to explore pathophysiology in patients and to examine novel treatments, Hogema and colleagues $^{54}$ ablated the murine Aldh5a1 gene. Although viable, this model may mimic the severe end of the phenotypic spectrum of ALDH5A1 deficiency, since it succumbs almost uniformly in lethal status epilepticus (SE) by 30 days of life. Seizure progression in these animals is temporally compressed, with absence seizures appearing at 14 days of life, tonic-clonic convulsions by 20 days of life and thereafter SE. ${ }^{55}$ GHB has been employed for a number of years for the induction of absence in rodents. ${ }^{13}$ Truncated lifespan provides a unique opportunity to explore pharmacotherapeutics to rescue the lethal phenotype. Chronic administration of the GHB receptor antagonist NCS-382, the $\mathrm{GABA}_{\mathrm{B}}$ receptor antagonist CGP-35348, the irreversible GABA transaminase inhibitor vigabatrin and taurine attenuate premature lethality. ${ }^{54}$ Results with NCS-382 are paradoxical, since there appears to be no functional alteration of the GHBergic system. ${ }^{56}$ Recent studies have revealed rescue of premature lethality employing the ketogenic diet, a mainstay for intervention in refractory epilepsy. ${ }^{49}$ Importantly, adenoviral constructs have rescued the mouse model from early lethality, providing proof-of-principle that hepatic correction may have utility in Aldh5a1 deficiency. ${ }^{57}$

Despite its severe neurological phenotype, the Aldh5a1-deficient mouse represents a faithful 
metabolic and neurochemical phenocopy of the human disorder. Analysis of regional brain sections has revealed significant accumulations of GABA, GHB, succinic semialdehyde (SSA) and several interrelated species (D-2-hydroxyglutarate [D-2-HG], 4,5dihydroxyhexanoic acid [DHHA], homocarnosine and guanidinobutyrate) ${ }^{58-62}$ All probably relate to metabolic by-products of GABA and GHB (see Figure 1), and their identification has provided new insights into disease pathophysiology. For example, recent studies have demonstrated that DHHA, pathognomonic for Aldh5a1 deficiency, is a weak ligand for the GHB receptor. ${ }^{62}$ The mouse model has also served as a roadmap for GABA receptor dysfunction in neural tissue. Snead and coworkers demonstrated that both $\mathrm{GABA}_{\mathrm{A}}$ and $\mathrm{GABA}_{\mathrm{B}}$ receptor-mediated neurotransmission is altered, with corresponding $\mathrm{GABA}_{\mathrm{A}}$ receptor subunit abnormalities, ${ }^{63,64}$ consistent with in vivo studies in patients (see above).

An inability to metabolise SSA to succinate raises the possibility that neurotoxic aldehydes might accumulate in tissues of affected individuals. Earlier work demonstrated that SSA accumulates in ALDH5A1-deficient mouse brain, ${ }^{58}$ but it remains likely that the majority of SSA is rapidly metabolised to DHHA or GHB (see Figure 1). Nonetheless, studies by Sauer and colleagues ${ }^{65}$ and Wajner and coworkers ${ }^{66-68}$ have provided evidence for oxidative stress in rodents administered GHB and in ALDH5A1-deficient mouse brain. This may be exacerbated by D-2-HG in the murine and human disorders. D-2-HG is proposed to derive from $\alpha$-ketoglutarate in a reaction by which the cell may attempt to detoxify accumulated GHB. This conversion is catalysed by an nicotinamide adenine dinucleotide $\left(\mathrm{NAD}^{+}\right)$-independent transhydrogenase, D-2-hydroxyglutarate transhydrogenase (HOT), first described by Kaufman and coworkers. ${ }^{69}$ Elevated D-2-HG is the biomarker in heritable D-2hydroxyglutaric aciduria, also with neurological sequelae. ${ }^{70}$ Depletion of $\alpha$-ketoglutarate from the tricarboxylic acid (TCA) cycle could lead to alterations in the intracellular NAD/NADH ratio, compounding the effects of oxidative stress. In neurones and glial cells, this would be further exacerbated by an inability to provide succinate residues via GABA catabolism, and compounded by the probable condensation of pyruvate (or lactate) with SSA to form DHHA (Figure 1). In addition, Murphy and coworkers ${ }^{11}$ demonstrated that ALDH5A1 is the major aldehyde dehydrogenase isozyme responsible for the metabolism of 4-HNE, a reactive aldehyde accumulating during membrane recycling which is linked to intracellular oxidant stress. ${ }^{71}$ It should be noted, however, that a number of redundant detoxification pathways for 4-HNE exist in almost all organs. The references cited above for $4-\mathrm{HNE}$ metabolism ${ }^{11,71}$ examined metabolic sequences in mitochondrial lysates lacking the cytosolic components (eg glutathione, glutathione transferase and other cytosolic aldehyde dehydrogenases) that may be important for oxidation of free aldehydes and aldehyde conjugates. It has been documented, however, that detoxification of 4-HNE in whole, intact brain mitochondria is primarily ALDH5A1 dependent. ${ }^{72,73}$

\section{Patterns of ALDH5AI alternative splicing}

The ALDH5A1 gene consists of ten exons encompassing over 38 kilobases $(\mathrm{kb})$. The complete open reading frame is 1,605 base pairs (bp) (accession number Y11192), encoding 535 amino acids (isoform 1), with the first 47 residues recognised as a mitochondrial targeting peptide. ${ }^{74}$ An 11 th exon, named $4 \mathrm{~B}$, is included between exons 4 and 5. Retention of exon $4 \mathrm{~B}$ into the transcript, owing to alternative splicing, produces the isoform 2 transcript variant, which putatively encodes for 548 amino acid residues. Qualitative assays reveal the presence of both isoforms in all human cell lines and tissues, but with different relative expression. Interindividual variation in the ratios of the two isoforms was detected in normal subjects by quantitative assays. It is currently unknown whether isoform 2 is translated into a functional polypeptide and its associated relative contribution. In vitro transfection of an isoform 2 cDNA construct produced an almost inactive enzyme. It remains to be determined if the product of isoform 2 could form mixed tetramers with that of isoform 1 . If so, an array of enzymes with possible differential activities 
might be produced, depending upon the exact tetramer composition. ${ }^{75}$

Additional heterogeneity of the ALDH5A1 transcript has been observed. Northern blot analysis of several tissues revealed the presence of two differentially expressed transcripts of approximately $5 \mathrm{~kb}$ and $2 \mathrm{~kb}$ in both human and rat. ${ }^{76}$ Analysis of the $3^{\prime}$ untranslated region (UTR) of the gene showed the presence of two polyadenylation signals whose alternative use accounts for two mRNAs of 1,827 and 5,225 nucleotides, respectively. The analysis of mRNA turnover suggested that the different $3^{\prime}$ UTR of the two transcripts was not responsible for differential stability or translation efficiency. Although no difference between the two mRNAs could be established, it cannot be excluded that differential properties could be restricted to particular physiological conditions and/or specific tissues. Different cis-acting elements in the $3^{\prime}$ UTR could be present in the two mRNAs and result in specific localisation in subcellular compartments. Along these lines, the higher level of the long transcript in brain could be attributed to a role of $3^{\prime}$ UTR sequences for the localisation in particular neuronal compartments. $^{75}$

\section{Common polymorphic variation in the ALDH5Al coding region}

ALDH5A1 activity measured in human lymphoblasts shows significant interindividual variability, with an approximately fivefold difference between the extremes. ${ }^{77}$ Several investigations were implemented to understand the basis for this variation. The analysis of the coding region in a panel of random healthy individuals identified eight missense variants and one same-sense variant, ${ }^{75}$ with four of them reaching polymorphic frequencies that is, c.106G $>\mathrm{C} \quad$ (p.G36R), c.538C $>\mathrm{T}$ (p.H180Y), c.545C $>$ T (p.P182L) and c.709G $>$ $\mathrm{T}$ (p.A273S). None of the identified polymorphic mutations affected exons 6 and 7, which encode the active site of the enzyme. In addition, all polymorphic missense variants, except c.709, targeted non-conserved residues.

To test the functional consequences of these missense mutations, variants were expressed in mammalian cells. The analysis revealed a wide spectrum of activities associated with each of the amino acid replacements, as compared with the most common form of the enzyme characterised by the highest value (Table 1). ${ }^{75}$ Among the amino acid changes occurring in the mature peptide, a significant activity reduction was observed for P182L. Conversely, the slight reduction displayed by the G36R variant could be attributed to altered mitochondrial targeting, as the amino acid change lies within the mitochondrial leader sequence. In vitro assays revealed that haplotypes with multiple amino acid replacements in the same polypeptide resulted in further reductions of activity, as compared with the single substitutions, ${ }^{40}$ possibly owing to a multiplicative effect. The finding of individuals heterozygous for several polymorphic positions prompted

Table I. ALDH5Al-coding polymorphisms

\begin{tabular}{|c|c|c|c|c|c|c|}
\hline $\begin{array}{l}\text { Gene } \\
\text { location } \\
\text { (exon) }\end{array}$ & dbSNP & $\begin{array}{l}\text { Position on } \\
\text { chromosome } \\
6 \text { (bp) }\end{array}$ & $\begin{array}{c}\text { Nucleotide } \\
\text { variation }\end{array}$ & $\begin{array}{l}\text { Amino acid } \\
\text { substitution }\end{array}$ & $\mathrm{MAF} \pm \mathrm{SE}$ & $\begin{array}{c}\text { Expressed } \\
\text { enzyme } \\
\text { activity* }\end{array}$ \\
\hline I & rs4646832 & 24603309 & c. $106 \mathrm{G}>\mathrm{C}$ & G36R & $0.051 \pm 0.036$ & $86.7 \%$ \\
\hline 3 & rs2760II8 & 24611569 & c. $538 \mathrm{C}>\mathrm{T}$ & HI80Y & $0.179 \pm 0.061$ & $82.5 \%$ \\
\hline 3 & rs3765310 & 24611576 & c.545C > T & PI82L & $0.051 \pm 0.036$ & $46.7 \%$ \\
\hline 4 & rs6262I664 & 24613175 & c.709G > T & A237S & $0.026 \pm 0.026$ & $65.1 \%$ \\
\hline 9 & rs61744005 & 2464037I & c. $1389 \mathrm{~T}>\mathrm{C}$ & D463D & $0.026 \pm 0.026$ & n.t. \\
\hline
\end{tabular}

* Expressed as a percentage of the corresponding wild-type control ( 100 per cent).

Abbreviations: bp, base pairs; dbSNP, Single Nucleotide Polymorphism database; MAF, minor allele frequency in Italian samples; n.t., not tested; SE, standard error 
Table 2. ALDH5AI haplotypes at five polymorphisms of the coding region

\begin{tabular}{|lccccc|}
$\begin{array}{l}\text { Coding } \\
\text { haplotype }\end{array}$ & c.106 & c.538 & c.545 & c.709 & c.1389 \\
\hline I & G & C & C & G & T \\
\hline 2 & G & C & C & T & T \\
\hline 3 & G & C & C & G & C \\
\hline 4 & G & T & C & G & T \\
\hline 5 & C & T & T & G & T \\
\hline
\end{tabular}

the analysis of the compound genotypes, to resolve the haplotype phase of the associated alleles.

Haplotype reconstruction for five coding positions (four missense and one same-sense) produced unequivocal results, with only five haplotypes of the 32 expected, and complete linkage disequilibrium (LD) between c.538T and both c.106C and c.545T alleles (Table 2) ${ }^{78}$ Therefore, the level of genotype diversity observed in humans predicted an array of enzymes, depending upon the subunit composition of the tetramer, with likely consequences on its overall catalytic activity. A more detailed view of the geographical pattern in the distribution of c.538 and c.545 coding variants was developed by genotyping subjects representing 60 populations from all continents. ${ }^{78,79}$ This analysis confirmed the presence of a restricted number of compound haplotypes and showed that the c.538C $>\mathrm{T}$ polymorphism is the most common: the c.538C allele shows a wide range of frequencies (from 0.50 to 1.0), with the lowest and highest extremes observed in African and Chinese populations, respectively. A high frequency of the haplotype carrying c.538T and c.545T was observed in all Asian populations, with a maximum represented by Papuan and Melanesian individuals (0.381 and 0.545, respectively).

The unusual pattern of human intraspecific diversity suggested a possible involvement of evolutionary processes and constraints that acted upon the ALDH5A1 gene. An evaluation of the extent of sequence divergence in terms of non-synonymous/ synonymous substitution rate $(d N / d S)$ was obtained by comparison with six primate coding sequences. None of the 25 non-synonymous substitutions found among primates altered any of the five amino acid motifs functionally important in the protein, thus confirming strong evolutionary constraints, already detected in the alignment of amino acids from largely divergent taxa. ${ }^{80}$ Strong conservation is consistent with the low $d N / d S$ ratio found among primates at the lower boundary of the distribution reported in extensive human-chimpanzee comparisons. By contrast, a $d N / d S$ ratio three times higher, displayed by the human lineage, raises the possibility of an acceleration in the rate of non-synonymous substitutions in the lineage leading to humans. Interspecific analyses enabled us to infer the ancestral state at all the variable coding positions identified in humans. In all but one polymorphism, the most common allele found in humans is represented by

Table 3. Eight single nucleotide polymorphism haplotypes of the ALDH5AI promoter region

\begin{tabular}{|ccccccccccc|}
$\begin{array}{l}\text { Promoter } \\
\text { haplotype }\end{array}$ & rsl6889393 & rsIl759284 & rs4646828 & rs2744575 & rs4646829 & rs7752835 & rs4646830 & rs4646831 & $\begin{array}{c}\text { Haplotype } \\
\text { accession } \\
\text { number }\end{array}$ \\
\hline I & G & T & C & G & C & G & G & G & AM943057 \\
\hline 2 & G & T & C & G & T & G & G & G & AM943056 \\
\hline 3 & n.t. & T & C & G & T & A & G & G & AM943059 \\
\hline 4 & G & T & C & C & C & G & C & G & AM943058 \\
\hline 5 & G & G & T & C & C & G & C & G & AM943055 \\
\hline 6 & A & T & C & G & C & G & G & A & AM943060 \\
\hline
\end{tabular}

Abbreviation: n.t., not tested 
the ancestral state, which is shared with primates. By contrast, the c.538C allele represents the derived allele, with very high frequencies in the majority of human populations, which is proceeding to replace the ancestral one. In particular, the c.538C allele causes the replacement of a tyrosine residue conserved in primates and rodents with a histidine never observed in other eukaryotes. The unexpected pattern of intraspecific allele distribution, compared with the interspecific findings, suggests the possibility of a recent positive selection that specifically acted in the human lineage, possibly by virtue of the advantage conferred by the maximal enzyme activity associated with the c.538C allele. ${ }^{78}$ Thus, human variation analysis has revealed an overall population differentiation and identified haplotypes with specific frequency distributions for $A L D H 5 A 1$. Consistent with these findings, a quota of the variation in enzyme activity detected in the general population can be attributed to the occurrence of missense mutations in a population-dependent manner.

\section{Polymorphic variants in the promoter region}

A further level of variation in the ALDH5A1 gene is demonstrated by single nucleotide polymorphisms (SNPs) detected in an $800 \mathrm{bp}$ sequence upstream of the ATG start codon and characterised by the presence of multiple promoter elements. ${ }^{75}$ Lorenz et al. ${ }^{10}$ identified seven variable positions in this region in three samples of patients and controls of German descent.

Resequencing of $870 \mathrm{bp}$ in 24 individuals with a broader range of geographical origins revealed polymorphism at nine positions, of which eight had been reported in the SNP DataBank (Table 3). Strong LD was observed within and between this region and the coding sequence, resulting in a limited number of promoter-coding arrangements. Haplotype reconstruction identified six arrangements for the eight variable promoter sites. To test the hypothesis that the different promoter haplotypes might influence transcriptional regulation, constructs in which these sequences had been cloned upstream of the luciferase gene were assayed. The results showed remarkable variation in the activity of the reporter gene and identified variants influencing transcriptional levels (Blasi et al.; manuscript in preparation). Two variant positions (rs4646828 and rs2744575) contribute significantly to the transcriptional level. This finding would confirm the presence of a regulatory positive element localised between -184 and -136 upstream of the $A L D H 5 A 1$ start codon. ${ }^{75}$ The higher diversity observed among the promoters associated with the c.538T coding allele, and the wider population distribution, are again consistent with its ancestral state.

\section{ALDH5AI variation in public databases}

A systematic exploration of diversity in the human genome is now feasible by accessing public databases. Six million validated SNPs have recently been reported (the National Center for Biotechnology Information, Single Nucleotide Polymorphism database [dbSNP], Build 127). An evaluation of the polymorphic level of the $A L D H 5 A 1$ region was obtained by exploring the last release of the dbSNP. A total of 270 SNPs was reported for a region of $43,986 \mathrm{bp}$, including $1,350 \mathrm{bp}$ upstream of the start codon and 3,930 downstream of the stop codon, although data on validation and population diversity are available for only about 50 per cent of these. To maximise the resolution of whole-genome association studies and limit the number of markers that require genotyping, the International HapMap project has generated dense genome-wide maps of SNPs and characterised haplotypes (combinations of SNPs in LD within a contiguous segment of DNA) from selected populations. Genotyping of selected world populations has allowed the construction of haplotype phases and frequencies relative to Europeans, Asians, Japanese and sub-Saharan Africans. All data are publicly available at http:// www.hapmap.org/cgi-perl/gbrowse/hapmap_B35/. The higher number of $A L D H 5 A 1$ haplotypes reported for sub-Saharan populations is consistent with a general increased level of genotypic diversity in comparison with other world populations. Several haplotypes show a population-specific pattern of distribution. It is still unknown whether some of these 
haplotypes might be associated with a phenotypic effect; further studies on specific populations could help in identifying possible phenotypes associated with the current level of $A L D H 5 A 1$ variation.

\section{ALDH5AI functional variants and susceptibility to quantitative traits}

In recent years, positional and functional evidence has suggested that $A L D H 5 A 1$ may be a candidate gene for some common quantitative traits. Evidence that functional ALDH5A1 activity is relevant to cognitive abilities derives from the observed symptomatology in patients affected by ALDH5A1 deficiency (see above), although it is not completely clear what level of decreased enzyme activity leads to induction of clinical symptoms. As ALDH5A1 deficiency is an autosomal recessive disorder, parents of patients are obligate heterozygotes. Enzyme studies in cells isolated from the blood of these individuals has generally revealed reduced activity, to approximately 50 per cent of normal, but no clinical findings on the whole. ${ }^{77}$ An exception to this was a report demonstrating EEG abnormalities in parents of ALDH5A1-affected individuals. ${ }^{81}$ The latter report raises the possibility that combinations of common variant alleles, occurring in coding and regulatory regions, could produce enzymes with suboptimal activity at a qualitative and/or quantitative level. Such alterations could alter GABA degradation regionally, resulting in metabolite accumulation (GHB and others; see Figure 1), which could alter neuronal excitability and lead to subclinical phenotypes.

The preceding hypothesis is supported by a potential link between ALDH5A1 and higher cognitive function. ${ }^{82}$ These investigators demonstrated that the c.538C allele was significantly associated with highintelligence quotient (IQ) individuals, as compared with average-IQ controls, accounting for a small effect of about 1.5 IQ points. Similarly, ALDH5A1 has been suggested as a possible positional candidate gene in developmental dyslexia or reading disability (RD) [OMIM 600202], based upon family linkage studies which identified $6 \mathrm{p} 21-22$ as one of the nine genomic regions showing peaks of linkage to the disorder. ${ }^{83,84}$ Single-marker and haplotype analyses in a group of families segregating for $\mathrm{RD}$ enabled the refinement of the candidate region for the $6 \mathrm{p}$ quantitative trait locus (QTL) influencing dyslexia. Single-marker association was found for five genes over an interval of about 3.4 megabases and spanning the $A L D H 5 A 1$ genomic region. These results were corroborated by the findings of haplotypes with significant over-transmission in probands selected for phenotypic severity. An association with a common three-marker haplotype in the $A L D H 5 A 1$ gene was also detected, ${ }^{83}$ potentially influenced by LD with the adjacent KIAA0319 and TTRAP genes strongly associated with $\mathrm{RD}{ }^{85}$

In order to determine the contribution of ALDH5A1 common coding and non-coding variants to disease risk, case-control association studies were performed in patients with idiopathic generalised epilepsy (IGE) and photosensitivity. ${ }^{10}$ These studies provided no evidence that any $A L D H 5 A 1$ missense variation, by itself, contributed to a common and substantial susceptibility effect in these phenotypes. A more exhaustive linkage study was performed by the multicentre collaborative group, the Epilepsy Genetics Consortium (EPIGEN) to assess whether common variants across selected candidate genes play a major role in the aetiology of epilepsy sub-phenotypes across multiple populations. Variations in ALDH5A1 and four other genes were most notable. The association with an ALDH5A1 intronic variant (rs2744601) produced one of the 25 significant $p$ values within individual populations. In particular, this SNP associated significantly with an absence phenotype in the Australian cohort $\left(p=3.33 \times 10^{-5}\right)$ and showed signs of association with generalised phenotypes correlated with absence, including generalised tonic-clonic seizures, juvenile myoclonic epilepsy (JME) and IGE non-JME, all within the same population. LD mapping closely to ALDH5A1-associated SNPs revealed two candidate-causal variants, both in exon 20 of the KIAA0319 gene. ${ }^{86}$ Although the authors did not identify clear, indisputable genetic factors associated with $A L D H 5 A 1$ and the other four genes, it remains possible that larger (and more syndromically focused) cohorts might reveal genetic variants with 


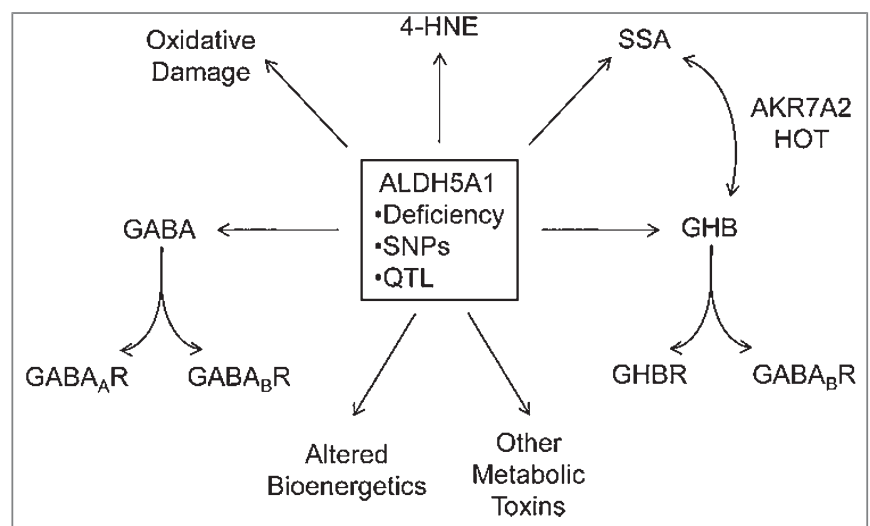

Figure 3. Proposed scheme highlighting concepts and principles reported in this review. The central box focuses on variation in the ALDH5AI gene, whether complete loss of activity or partial alteration linked to single nucleotide polymorphism (SNP), splicing variation or other features. The outward arrows reveal the potential results of these nucleic acid alterations, including metabolic findings (altered levels of $\gamma$-aminobutyric acid [GABA], $\gamma$-hydroxybutyrate [GHB], succinate semialdehyde [SSA] and 4-hydroxy-2-nonenal [4-HNE]), processes (receptor stimulation and/or downregulation, including $G A B A_{A}$ receptor $\left[G A B A_{A} R\right.$ ], GABA receptor $\left[G A B A_{B} R\right]$ and $G H B$ receptor $[G H B R]$ ) and other processes (eg oxidative stress, altered bioenergetics and the accumulation of other metabolic toxins whose role in pathophysiology remains to be clarified). Additional abbreviations: HOT, D-2-hydroxyglutarate transhydrogenase; AKR7A2, aldo-keto reductase 7A2; QTL, quantitative trait loci.

weak effects and confirmation of an apparent population-specific genetic risk.

The potential contribution of an ALDH5A1 enzyme with decreased catalytic activity to the induction of oxidative stress was evaluated by studying the association between the functional c.538C > $\mathrm{T}$ polymorphism and the preservation of cognitive function and survival in an Italian sample (514 subjects, 18-107 years of age). ${ }^{9}$ In relation to the reduced activity of the $\mathrm{Tyr}_{180}$ form of the enzyme (corresponding to the $\mathrm{T}$ allele) and its association with cognitive performance across the general population, ${ }^{82}$ the authors tested the hypothesis that c.538C $>\mathrm{T}$ polymorphisms could play a role in cognitive decline variation, which characterises subjects over 65 years. The study examined specific tests for cognitive assessment and employed genotyping for the ALDH5A1 polymorphism. The results revealed that, within the $65-85$ year range, the $\mathrm{T} / \mathrm{T}$ homozygous genotype is over-represented in subjects with impaired cognitive function (Mini-Mental Status Examination [MMSE] $\leq 23$ ) compared with those with conserved cognitive function (MMSE > 23). In addition, the $T / T$ genotype affects survival beyond 65 years of age; in fact, after this age, the survival fraction of $\mathrm{T} / \mathrm{T}$ subjects is significantly lower than $\mathrm{C} / \mathrm{T}$ or $\mathrm{C} / \mathrm{C}$ individuals. Although the association between the c.538C $>\mathrm{T}$ polymorphism and the preservation of cognitive function requires confirmation in a larger sample, these data suggest that reduced ALDH5A1 catalytic activity may induce oxidative stress, which is crucial in modulating the rate and the quality of ageing.

\section{Conclusion}

ALDH5A1 appears to be acquiring a more important role, both as a GABA catabolic enzyme and potentially as a detoxifying enzyme involved in aldehyde scavenging. Perhaps most importantly, growing evidence suggests that $A L D H 5 A 1$ SNPs may have a role in a number of quantitative traits (Figure 3). Expanded population-based studies, and additional characterisation of ALDH5A1-deficient patients and mice, will assuredly reveal additional facets of the comparative genomic features of ALDH5A1, and its importance in normal CNS function.

\section{Acknowledgments}

The authors are indebted to a large number of colleagues who have been instrumental in studies on SSADH-deficient patients and mice, including Erwin Jansen, and Drs Eduard Struys, Gajja Salomons, Kirk Nylen, Miguel Cortez, Jose Luis Perez Velazquez, Andrea Buzzi, Ying Wu and Phillip Pearl. The financial support of NIH NS40270 and the Pediatric Neurotransmitter Disease Association is also gratefully acknowledged.

\section{References}

1. Hedou, G., Chasserot-Golaz, S., Kemmel, V. et al. (2000), 'Immunohistochemical studies of the localization of neurons containing the enzyme that synthesizes dopamine, GABA, or gammahydroxybutyrate in the rat substantia nigra and striatum', J. Comp. Neurol. Vol. 426, pp. 549-560.

2. Obata, K., Hirono, M., Kume, N., Kawaguchi, Y., Itohara, S. and Yanagawa, Y. (2008), 'GABA and synaptic inhibition of mouse cerebellum lacking glutamate decarboxylase 67', Biochem. Biophys. Res. Commun. Vol. 370, pp. 429-433. 
3. Jaeken, J., Casaer, P., de Cock, P. et al. (1984), 'Gamma-aminobutyric acid-transaminase deficiency: A newly recognized inborn error of neurotransmitter metabolism', Neuropediatrics Vol. 15, pp. 165-169.

4. Jakobs, C., Bojasch, M., Mönch, E., Rating, D., Siemes, H. and Hanefeld, F. (1981), 'Urinary excretion of gamma-hydroxybutyric acid in a patient with neurological abnormalities. The probability of a new inborn error of metabolism', Clin. Chim. Acta Vol. 111, pp. $169-178$

5. Pearl, P.L., Gibson, K.M., Acosta, M.T. et al. (2003), 'Clinical spectrum of succinic semialdehyde dehydrogenase deficiency', Neurology Vol. 60, pp. 1413-1417

6. Pearl, P.L., Taylor, J.L., Trzcinski, S. and Sokohl, A. (2007), 'The pediatric neurotransmitter disorders', J. Child Neurol. Vol. 22, pp. 606-616.

7. Bray, N.J., Holmans, P.A., van den Bree, M.B. et al. (2008), 'Cis- and trans- loci influence expression of the schizophrenia susceptibility gene DTNBP1', Hum. Mol. Genet. Vol. 17, pp. 1169-1174.

8. Brkanac, Z., Chapman, N.H., Matsushita, M.M. et al. (2007), 'Evaluation of candidate genes for DYX1 and DYX2 in families with dyslexia', Am. J. Med. Genet. Part B: Neuropsychiatric Genetics Vol. 144, pp. $556-560$.

9. De Rango, F., Leone, O., Dato, S. et al. (2008), 'Cognitive functioning and survival in the elderly: The SSADH C538T polymorphism', Ann. Hum. Genet. Vol. 72, pp. 630-635.

10. Lorenz, S., Heils, A., Taylor, K.P. et al. (2006), 'Candidate gene analysis of the succinic semialdehyde dehydrogenase gene (ALDH5A1) in patients with idiopathic generalized epilepsy and photosensitivity', Neurosci. Lett. Vol. 397, pp. 234-239.

11. Murphy, T.C., Amarnath, V., Gibson, K.M. and Picklo, M.J. Sr. (2003), 'Oxidation of 4-hydroxy-2-nonenal by succinic semialdehyde dehydrogenase (ALDH5A1)', J. Neurochem. Vol. 86, pp. 298-305.

12. Snead, O.C. III and Gibson, K.M. (2005), 'Gamma-hydroxybutyric acid', N. Engl. J. Med. Vol. 352, pp. 2721-2732.

13. Liu, Z., Snead, O.C. III, Vergnes, M., Depaulis, A. and Marescaux, C. (1991), 'Intrathalamic injections of gamma-hydroxybutyric acid increase genetic absence seizures in rats', Neurosci. Lett. Vol. 125, pp. $19-21$.

14. Cash, C.D. (1994), 'Gamma-hydroxybutyrate: An overview of the pros and cons for it being a neurotransmitter and/or a useful therapeutic agent', Neurosii. Biobehav. Rev. Vol. 18, pp. 291-304.

15. Maitre, M. (1997), 'The gamma-hydroxybutyrate signalling system in brain: Organization and functional implications', Prog. Neurobiol. Vol. 51, pp. $337-361$

16. Snead, O.C. III and Bearden, L.J. (1980), 'Naloxone overcomes the dopaminergic, EEG, and behavioral effects of gamma-hydroxybutyrate', Neurology Vol. 30, pp. 832-838.

17. Dyer, J.E. (1991), 'Gamma-hydroxybutyrate: A health-food product producing coma and seizure-like activity', Am. J. Emerg. Med. Vol. 9, pp. 321-324.

18. Smith, K.M. (1999), 'Drugs used in acquaintance rape', J. Am. Pharm. Assoc. Vol. 39, pp. 519-525.

19. Xyrem International Study Group. (2005), 'A double-blind, placebocontrolled study demonstrates sodium oxybate is effective for the treatment of excessive daytime sleepiness in narcolepsy', J. Clin. Sleep Med. Vol. 15, pp. 391-397.

20. Nava, F., Premi, S., Manzato, E., Campagnola, W., Lucchini, A. and Gessa, G.L. (2007), 'Gamma-hydroxybutyrate reduces both withdrawal syndrome and hypercortisolism in severe abstinent alcoholics: An open study vs. diazepam', Am. J. Drug Alcohol Abuse Vol. 33, pp. 379-392.

21. Scharf, M.B., Baumann, M. and Berkowitz, D.V. (2003), 'The effects of sodium oxybate on clinical symptoms and sleep patterns in patients with fibromyalgia', J. Rheumatol. Vol. 30, pp. 1070-1074.

22. Schwartz, T.L. (2007), 'Gamma hydroxy butyric acid and sodium oxybate used to treat posttraumatic stress disorder', CNS Spectrums Vol. 12, pp. 884-886.

23. Frucht, S.J., Houghton, W.C., Bordelon, Y., Greene, P.E. and Louis, E.D. (2005), 'A single-blind, open-label trial of sodium oxybate for myoclonus and essential tremor', Neurology Vol. 65, pp. 1967-1969.
24. Kaupmann, K., Cryan, J.F., Wellendorph, P. et al. (2003), 'Specific gamma-hydroxybutyrate-binding sites but loss of pharmacological effects of gamma-hydroxybutyrate in GABA(B)(1)-deficient mice', Eur. J. Neurosci. Vol. 18, pp. 2722-2730.

25. Lingenhoehl, K., Brom, R., Heid, J. et al. (1999), 'Gammahydroxybutyrate is a weak agonist at recombinant $\mathrm{GABA}(\mathrm{B})$ receptors', Neuropharmacology Vol. 38, pp. 1667-1673.

26. Snead, O.C. III and Liu, C.C. (1984), 'Gamma-hydroxybutyric acid binding sites in rat and human brain synaptosomal membranes', Biochem. Pharmacol. Vol. 33, pp. 2587-2590.

27. Snead, O.C. III (1984), 'Ontogeny of gamma-hydroxybutyric acid. II. Electroencephalographic effects', Brain Res. Vol. 317, pp. 89-96.

28. Castelli, M.P., Mocci, I., Langlois, X. et al. (2000), 'Quantitative autoradiographic distribution of gamma-hydroxybutyric acid binding sites in human and monkey brain', Brain Research, Mol. Brain Res. Vol. 78, pp. 91-99.

29. Gould, G.G., Mehta, A.K., Frazer, A. and Ticku, M.K. (2003), 'Quantitative autoradiographic analysis of the new radioligand $[(3) \mathrm{H}](2 \mathrm{E})-$ (5-hydroxy-5,7,8,9-tetrahydro-6H-benzo[a][7]annulen-6-ylidene) ethanoic acid $([(3) \mathrm{H}] \mathrm{NCS}-382)$ at gamma-hydroxybutyric acid (GHB) binding sites in rat brain', Brain Res. Vol. 979, pp. 51-56.

30. Murphy, T.C., Poppe, C., Porter, J.E., Montine, T.J. and Picklo, M.J. Sr. (2004), '4-Hydroxy-trans-2-nonenoic acid is a gamma-hydroxybutyrate receptor ligand in the cerebral cortex and hippocampus', J. Neurochem. Vol. 89 , pp. $1462-1470$.

31. Andriamampandry, C., Taleb, O., Viry, S et al. (2003), 'Cloning and characterization of a rat brain receptor that binds the endogenous neuromodulator gamma-hydroxybutyrate (GHB)', FASEB J. Vol. 17, pp. 1691-1693.

32. Andriamampandry, C., Taleb, O., Kemmel,, V., Humbert, J.P., Aunis, D. and Maitre, M. (2007), 'Cloning and functional characterization of a gamma-hydroxybutyrate receptor identified in the human brain', FASEB J. Vol. 21, pp. 885-895.

33. Kemmel, V., Miehe, M., Roussel, G. et al. (2006), 'Immunohistochemical localization of a $\mathrm{GHB}$ receptor-like protein isolated from rat brain', $J$. Comp. Neurol. Vol. 498, pp. 508-524.

34. Snead, O.C. III and Nichols, A.C. (1987), 'Gamma-hydroxybutyric acid binding sites: Evidence for coupling to a chloride anion channel', Neuropharmacology Vol. 26, pp. 1519-1523.

35. Eli, M. and Cattabeni, F. (1983), 'Endogenous gamma-hydroxybutyrate in rat brain areas: Postmortem changes and effects of drugs interfering with gamma-aminobutyric acid metabolism', J. Neurochem. Vol. 41, pp. $524-530$.

36. Lyon, R.C., Johnston, S.M., Watson, D.G., McGarvie, G. and Ellis, E.M. (2007), 'Synthesis and catabolism of gamma-hydroxybutyrate in SH-SY5Y human neuroblastoma cells: Role of the aldo-keto reductase AKR7A2', J. Biol. Chem. Vol. 282, pp. 25986-25992.

37. Picklo, M.J. Sr., Olson, S.J., Hayes, J.D., Markesbery, W.R. and Montine, T.J. (2001), 'Elevation of AKR7A2 (succinic semialdehyde reductase) in neurodegenerative disease', Brain Res. Vol. 916, pp. 229-238.

38. Ando, N., Gold, B.I., Bird, E.D. and Roth, R.H. (1979), 'Regional brain levels of gamma-hydroxybutyrate in Huntington's disease', $J$. Neurochem. Vol. 32, pp. 617-622.

39. Ando, N., Simon, J.R. and Roth, R.H. (1979), 'Inverse relationship between GABA and gamma-hydroxybutyrate levels in striatum of rat injected with kainic acid', J. Neurochem. Vol. 32, pp. 623-625.

40. Akaboshi, S., Hogema, B.M., Novelletto, A. et al. (2003), 'Mutational spectrum of the succinate semialdehyde dehydrogenase (ALDH5A1) gene and functional analysis of 27 novel disease-causing mutations in patients with SSADH deficiency', Hum. Mutat. Vol. 22, pp. 442-450.

41. Vasiliou, V. and Nebert, D.W. (2005), 'Analysis and update of the human aldehyde dehydrogenase (ALDH) gene family', Hum. Genom. Vol. 2, pp. $138-143$.

42. Bekri, S., Fossoud, C., Plaza, G. et al. (2004), 'The molecular basis of succinic semialdehyde dehydrogenase deficiency in one family', Mol. Genet. Metab. Vol. 81, pp. 347-351.

43. Lemes, A., Blasi, P., Gonzales, G. et al. (2006), 'Succinic semialdehyde dehydrogenase (SSADH) deficiency: Molecular analysis in a South American family', J. Inherit. Metab. Dis. Vol. 29, p. 587. 
44. Blasi, P., Palmerio, F., Caldarola, S. et al. (2006), 'Succinic semialdehyde dehydrogenase deficiency: Clinical, biochemical and molecular characterization of a new patient with severe phenotype and a novel mutation', Clin. Genet. Vol. 69, pp. 294-296.

45. Jung, R., Rauch, A., Salomons, G.S. et al. (2006), 'Clinical, cytogenetic and molecular characterization of a patient with combined succinic semialdehyde dehydrogenase deficiency and incomplete WAGR syndrome with obesity', Mol. Genet. Metab. Vol. 88, pp. 256-260.

46. Knerr, I., Gibson, K.M., Jakobs, C. and Pearl, P.L. (2008), 'Neuropsychiatric morbidity in adolescent and adult succinic semialdehyde dehydrogenase (SSADH) deficiency', CNS Spectrums, Vol. 13, pp. 598-605.

47. Gropman, A. (2003), 'Vigabatrin and newer interventions in succinic semialdehyde dehydrogenase deficiency', Ann. Neurol. Vol. 54 (Suppl. 6), pp. S66-S72.

48. Leuzzi, V., Di Sabato, M.L., Deodato, F. et al. (2007), 'Vigabatrin improves paroxysmal dystonia in succinic semialdehyde dehydrogenase deficiency', Neurology Vol. 68, pp. 1320-1321.

49. Nylen, K., Velazquez, J.L., Likhodii, S.S. et al. (2008), 'A ketogenic diet rescues the murine succinic semialdehyde dehydrogenase deficient phenotype', Exp. Neurol. Vol. 210, pp. 449-457.

50. Saronwala, A., Tournay, A. and Gargus, J.J. (2008), 'Taurine treatment of succinate semialdehyde dehydrogenase (SSADH) deficiency reverses MRI-documented globus lesion and clinical syndrome', American College of Medical Genetics, Annual Meeting, 12-16 March, Phoeniz, AZ.

51. Gibson, K.M., Gupta, M., Pearl, P.L. et al. (2003), 'Significant behavioral disturbances in succinic semialdehyde dehydrogenase (SSADH) deficiency (gamma-hydroxybutyric aciduria)', Biol. Psychiatry Vol. 54, pp. 763-768.

52. Pearl, P.L., Taylor, J., Trzcinski, S. et al. (2007), '11C - Flumazenil PET imaging in patients with SSADH deficiency'. Program No. 2.140. 2007 Abstract Viewer. American Epilepsy Society, Philadelphia, PA, available at: www.aesnet.org/go/publications/aes-abstracts/abstract-search/?mode= display\&st=flumazenil $\% 20 \mathrm{PET} \% 20$ imaging\&sy $=2007 \& \mathrm{sb}=$ All\&startrow $=$ 18 id $=7589$.

53. Reis, J., Cohen, L.G., Pearl, P.L., Gibson, K.M., Dustin, I. and Theodore, W.H. (2007), 'Transcranial magnetic stimulation reveals altered cortical excitability in succinic semialdehyde dehydrogenase deficiency'. Program No. 3.029. 2007 Abstract Viewer. American Epilepsy Society, Philadelphia, PA, available at: www.aesnet.org/go/publications/aesabstracts $/$ abstract-search $/$ ?mode $=$ display\&st $=$ transcranial $\% 20$ magnetic $\% 20$ stimulation $\& \mathrm{sy}=2007 \& \mathrm{sb}=$ All $\&$ startrow $=1 \& \mathrm{Rid}=7775$.

54. Hogema, B.M., Gupta, M., Senephansiri, H. et al. (2001), 'Pharmacologic rescue of lethal seizures in mice deficient in succinate semialdehyde dehydrogenase', Nat. Genet. Vol. 29, pp. 212-216.

55. Cortez, M.A., Wu, Y., Gibson, K.M. and Snead, O.C. III (2004), 'Absence seizures in succinic semialdehyde dehydrogenase deficient mice: A model of juvenile absence epilepsy', Pharmacol. Biochem. Behav. Vol. 79, pp. 547-53

56. Mehta, A.K., Gould, G.G., Gupta, M., Carter, L.P., Gibson, K.M. and Ticku, M.K. (2006), 'Succinate semialdehyde dehydrogenase deficiency does not down-regulate gamma-hydroxybutyric acid binding sites in the mouse brain', Mol. Genet. Metab. Vol. 88, pp. 86-89.

57. Gupta, M., Jansen, E.E., Senephansiri, H. et al. (2004), 'Liver-directed adenoviral gene transfer in murine succinate semialdehyde dehydrogenase deficiency', Mol. Ther. Vol. 9, pp. 527-539.

58. Gibson, K.M., Gupta, M., Senephansiri, H. et al. (2006), 'Oxidant stress and neurodegeneration in murine succinic semialdehyde dehydrogenase (SSADH) deficiency', in: Hoffmann, G.F. (ed.), 'Diseases of Neurotransmission: From Bench to Bed', SPS Verlagsgessellschaft mbH, Heilbronn, Germany, Symposia Proceedings, pp. 199-212.

59. Jansen, E.E., Verhoeven, N.M., Jakobs, C. et al. (2006), 'Increased guanidino species in murine and human succinate semialdehyde dehydrogenase (SSADH) deficiency', Biochim. Biophys. Acta Vol. 1762, pp. 494-498.

60. Jansen, E.E., Gibson, K.M., Shigematsu, Y., Jakobs, C. and Verhoeven, N.M. (2006), 'A novel, quantitative assay for homocarnosine in cerebrospinal fluid using stable-isotope dilution liquid chromatographytandem mass spectrometry', J. Chromatogr. B Analyt. Technol. Biomed. Life Sci. Vol. 830, pp. 196-200.
61. Struys, E.A., Jansen, E.E., Gibson, K.M. and Jakobs, C. (2005), 'Determination of the GABA analogue succinic semialdehyde in urine and cerebrospinal fluid by dinitrophenylhydrazine derivatization and liquid chromatography-tandem mass spectrometry: Application to SSADH deficiency'. J. Inherit. Metab. Dis. Vol. 28, pp. 913-920.

62. Struys, E.A., Verhoeven, N.M., Jansen, E.E. et al. (2006), 'Metabolism of gamma-hydroxybutyrate to d-2-hydroxyglutarate in mammals: Further evidence for d-2-hydroxyglutarate transhydrogenase', Metabolism Vol. 55, pp. 353-358.

63. Buzzi, A., Wu, Y., Frantseva, M.V. et al. (2006), 'Succinic semialdehyde dehydrogenase deficiency: GABAB receptor-mediated function', Brain Res. Vol. 1090, pp. 15-22.

64. Wu, Y., Buzzi, A., Frantseva, M. et al. (2006), 'Status epilepticus in mice deficient for succinate semialdehyde dehydrogenase: GABAA receptormediated mechanisms', Ann. Neurol. Vol. 59, pp. 42-52.

65. Sauer, S.W., Kölker, S., Hoffmann, G.F. et al. (2007), 'Enzymatic and metabolic evidence for a region specific mitochondrial dysfunction in brains of murine succinic semialdehyde dehydrogenase deficiency (Aldh5a1-/-mice)', Neurochem. Int. Vol. 50, pp. 653-659.

66. Dutra-Filho, C.S., Wajner, M., Wannmacher, C.M. et al. (1995), 'Inhibition of citrate oxidation in vitro by 2-hydroxybutyrate and 4hydroxybutyrate in cerebral cortex of young rats', Biochem. Soc. Trans. Vol. 23, p. 229S

67. Dutra-Filho, C.S., Wajner, M., Wannmacher, C.M. et al. (1995), '2Hydroxybutyrate and 4-hydroxybutyrate inhibit $\mathrm{CO} 2$ formation from labeled substrates by rat cerebral cortex', Biochem. Soc. Trans. Vol. 23, p. $228 \mathrm{~S}$.

68. Latini, A., Scussiato, K., Leipnitz, G., Gibson, K.M. and Wajner, M. (2007), 'Evidence for oxidative stress in tissues derived from succinate semialdehyde dehydrogenase-deficient mice', J. Inherit. Metab. Dis. Vol. 30, pp. 800-810.

69. Kaufman, E.E., Nelson, T., Fales, H.M. and Levin, D.M. (1988), 'Isolation and characterization of a hydroxyacid-oxoacid transhydrogenase from rat kidney mitochondria', J. Biol. Chem. Vol. 263, pp. 16872-16879.

70. Struys, E.A. (2006), 'D-2-Hydroxyglutaric aciduria: Unravelling the biochemical pathway and the genetic defect', J. Inherit. Metab. Dis. Vol. 29, pp. $21-29$.

71. Reed, T., Perluigi, M., Sultana, R. et al. (2008), 'Redox proteomic identification of 4-hydroxy-2-nonenal-modified brain proteins in amnestic mild cognitive impairment: Insight into the role of lipid peroxidation in the progression and pathogenesis of Alzheimer's disease', Neurobiol. Dis. Vol. 30, pp. 107-120.

72. Honzatko, A., Brichac, J., Murphy, T.C. et al. (2005), 'Enantioselective metabolism of trans-4-hydroxy-2-nonenal by brain mitochondria', Free Radic. Biol. Med. Vol. 39, pp. 913-924.

73. Meyer, M.J., Mosely, D.M. and Picklo, M.J. (2004), 'Metabolism of 4hydroxy-trans-2-nonenal by CNS mitochondria is dependent on age and $\mathrm{NAD}^{+}$availability', Chem. Res. Toxicol. Vol. 17, pp. 1272-1279.

74. Chambliss, K.L., Hinson, D.D., Trettel, F. et al. (1998), 'Two exon-skipping mutations as the molecular basis of succinic semialdehyde dehydrogenase deficiency (4-hydroxybutyric aciduria)', Am. J. Hum. Genet. Vol. 63, pp. 399-408.

75. Blasi, P., Boyl, P.P., Ledda, M. et al. (2002), 'Structure of human succinic semialdehyde dehydrogenase gene: Identification of promoter region and alternatively processed isoforms', Mol. Genet. Metab. Vol. 76, pp. 348-362.

76. Chambliss, K.L., Zhang, Y.A., Rossier, E., Vollmer, B. and Gibson, K.M. (1995), 'Enzymatic and immunologic identification of succinic semialdehyde dehydrogenase in rat and human neural and nonneural tissues', J. Neurochem. Vol. 65, pp. 851-855.

77. Gibson, K.M., Lee, C.F, Chambliss, K.L. et al. (1991), '4-Hydroxybutyric aciduria: Application of a fluorometric assay to the determination of SSA activity in extracts of cultured human lymphoblasts', Clin. Chim. Acta Vol. 196, pp. 219-221.

78. Blasi, P., Palmerio, F., Aiello, A., Rocchi, M., Malaspina, P. and Novelletto, A. (2006), 'SSADH variation in primates: Intra and inter specific data on a gene with a potential role in human cognitive functions', J. Mol. Evol. Vol. 63, pp. 54-68. 
79. Leone, O., Blasi, P., Palmerio, F., Kozlov, A.I., Malaspina, P. and Novelletto, A. (2006), 'A human derived SSADH coding variant is replacing the ancestral allele shared with primates', Ann. Hum. Biol. Vol. 33, pp. 593-603.

80. Busch, K.B. and Fromm, H. (1999), 'Plant succinic semialdehyde dehydrogenase. Cloning, purification, localization in mitochondria, and regulation by adenine nucleotides', Plant Physiol. Vol. 21, pp. $589-597$.

81. Dervent, A., Gibson, K.M., Pearl, P.L., Salomons, G.S., Jakobs, C. and Yalcinkaya, C. (2004), 'Photosensitive absence epilepsy with myoclonias and heterozygosity for succinic semialdehyde dehydrogenase (SSADH) deficiency', Clin. Neurophysiol. Vol. 115, pp. 1417-1422.

82. Plomin, R., Turic, D.M., Hill, L. et al. (2004), 'A functional polymorphism in the succinate-semialdehyde dehydrogenase (aldehyde dehydrogenase 5 family, member A1) gene is associated with cognitive ability', Mol. Psychiatry Vol. 9, pp. 582-586.

83. Deffenbacher, K.E., Kenyon, J.B., Hoover, D.M. et al. (2004), 'Refinement of the $6 \mathrm{p} 21.3$ quantitative trait locus influencing dyslexia: Linkage and association analyses', Hum. Genet. Vol. 115, pp. 128-138.

84. Fisher, S.E. and DeFries, J.C. (2002), 'Developmental dyslexia: Genetic dissection of a complex cognitive trait', Nat. Rev. Neurosci. Vol. 3, pp. 767-780.

85. Luciano, M., Lind, P.A., Duffy, D.L. et al. (2007), 'A haplotype spanning KIAA0319 and TTRAP is associated with normal variation in reading and spelling ability', Biol. Psychiatry Vol. 62, pp. 811-817.

86. Cavalleri, G.L., Weale, M.E., Shianna, K.V. et al. (2007), 'Multicentre search for genetic susceptibility loci in sporadic epilepsy syndrome and seizure types: a case-control study', Lancet Neurol. Vol. 6, pp. 970-980. 